\title{
A NOTE ON DUAL EQUATIONS WITH TRIGONOMETRICAL KERNELS
}

\author{
by C. J. TRANTER \\ (Received 16th February 1963)
}

1. Sneddon (1) has recently pointed out that the dual integral equations

$$
\begin{array}{ll}
\int_{0}^{\infty} \xi^{-1} \psi(\xi) \cos (x \xi) d \xi=f(x) & (0 \leqq x \leqq 1), \\
\int_{0}^{\infty} \psi(\xi) \cos (x \xi) d \xi=0 & (x>1), \ldots .
\end{array}
$$

are not covered by Busbridge's solution (2) of the more general equations with Bessel function kernels and he has given a solution to equations (1) and (2) in the form of a Neumann series. The purpose of this note is to show that a very simple formal solution can be obtained by using the well-known Bessel function integral representations ((3), pp. 48 and 170)

$$
\frac{\pi}{2} J_{0}(r \xi)=\int_{0}^{r} \frac{\cos (\xi x) d x}{\left(r^{2}-x^{2}\right)^{\frac{1}{2}}}=\int_{r}^{\infty} \frac{\sin (\xi x) d x}{\left(x^{2}-r^{2}\right)^{\frac{1}{2}}}
$$

The similar problem in which $\sin (x \xi)$ replaces $\cos (x \xi)$ in equations (1) and (2) is also solved in the same way.

2. We start by integrating equation (2) with respect to $x$ to give

$$
\int_{0}^{\infty} \xi^{-1} \psi(\xi) \sin (x \zeta) d \xi=C \text { (constant) } \quad(x>1)
$$

As $x \rightarrow \infty$, the value of this integral tends to $\frac{1}{2} \pi \psi(+0)$ and, since $\psi(+0)=0$ if the integral in (1) is to exist, it follows that $C=0$ and equation (4) becomes

$$
\int_{0}^{\infty} \xi^{-1} \psi(\xi) \sin (x \xi) d \xi=0 \quad(x>1)
$$

Multiplication of equations (1) and (5) respectively by $\left(r^{2}-x^{2}\right)^{-\frac{1}{2}}$ and $\left(x^{2}-r^{2}\right)^{-\frac{1}{2}}$, integration with respect to $x$ between $0, r$ and $r, \infty$, and use of the integral representations (3) leads to

$$
\frac{\pi}{2} \int_{0}^{\infty} \xi^{-1} \psi(\xi) J_{0}(r \xi) d \xi= \begin{cases}\int_{0}^{r} \frac{f(x) d x}{\left(r^{2}-x^{2}\right)^{\frac{1}{2}}} & (0<r<1), \\ 0 & (r>1) .\end{cases}
$$


Application of the Hankel inversion theorem then gives

$$
\frac{\pi}{2} \xi^{-2} \psi(\xi)=\int_{0}^{1} r J_{0}(\ddot{\zeta} r) d r \int_{0}^{r} \frac{f(x) d x}{\left(r^{2}-x^{2}\right)^{\frac{1}{2}}} .
$$

3. In the solution of the dual equations

$$
\begin{aligned}
& \int_{0}^{\infty} \xi^{-1} \psi(\xi) \sin (x \xi) d \xi=f(x) \quad(0 \leqq x \leqq 1), \\
& \int_{0}^{\infty} \psi(\xi) \sin (x \xi) d \xi=0 \quad(x>1),
\end{aligned}
$$

we first differentiate equation (8) to give

$$
\int_{0}^{\infty} \psi(\xi) \cos (x \xi) d \xi=f^{\prime}(x) \quad(0 \leqq x \leqq 1) .
$$

Using the same procedure on equations (10) and (9) as was used with equations (1) and (5) we find

$$
\frac{\pi}{2} \int_{0}^{\infty} \psi(\xi) J_{0}(r \xi) d \xi=\left\{\begin{array}{cl}
\int_{0}^{r} \frac{f^{\prime}(x) d x}{\left(r^{2}-x^{2}\right)^{\frac{1}{2}}} & (0<r<1), \\
0 & (r>1),
\end{array}\right.
$$

so that, in this case,

$$
\frac{\pi}{2} \xi^{-1} \psi(\xi)=\int_{0}^{1} r J_{0}(\xi r) d r \int_{0}^{r} \frac{f^{\prime}(x) d x}{\left(r^{2}-x^{2}\right)^{\frac{1}{2}}} .
$$

\section{REFERENCES}

(1) I. N. Sneddon, Proc. Glasgow Math. Assoc., 5 (1962), 147-152.

(2) I. W. Busbridge, Proc. London Math. Soc., 44 (1938), 115-129.

(3) G. N. Watson, Theory of Bessel functions (second edition, Cambridge University Press, 1944).

Royal Military College of Science

SHRIVENHAM 